\title{
Hemodynamics and Flow Characteristics of a New Dialysis Port
}

\author{
Jérémie Guignard, *† Muluken Behran Terefe,* Dominik E. Uehlinger,‡ and Justyna Czerwinska*‡
}

\begin{abstract}
Renal replacement therapy by hemodialysis requires a permanent vascular access. Implantable ports offer a potential alternative to standard vascular access strategies although their development is limited both in number and extent. We explored the fluid dynamics within two new percutaneous bone-anchored dialysis port prototypes, both by in vitro experiments and computer simulation. The new port is to be fixed to bone and allows the connection of a dialysis machine to a central venous catheter via a built-in valve. We found that the pressure drop induced by the two ports was between 20 and $50 \mathrm{mmHg}$ at $500 \mathrm{ml} / \mathrm{min}$, which is comparable with commercial catheter connectors $(15-80 \mathrm{mmHg})$. We observed the formation of vortices in both geometries, and a shear rate in the physiological range $\left(<10,000 \mathrm{~s}^{-1}\right)$, which is lower than maximal shear rates reported in commercial catheters (up to $13,000 \mathrm{~s}^{-1}$ ). A difference in surface shear rate of $15 \%$ between the two ports was obtained. ASAIO Journal 2014; 60:81-89.
\end{abstract}

Key Words: dialysis port, hemodynamics, catheter, finite elements model

$\mathrm{H}$ emodialysis is the most widely used renal replacement procedure in patients with end-stage renal disease (ESRD). To be able to perform the necessary three dialysis treatment sessions per week, a permanent vascular access is mandatory. Today, the preferred vascular access is the surgical construction of a peripheral arteriovenous (AV) fistula and if this is not possible, of a synthetic graft. However, the high age of the population on dialysis with a median age in Switzerland currently above 70 years and the polymorbidity of ESRD patients often preclude the construction of an AV-fistula and permanent tunneled central venous catheters (CVCs) are placed instead.

From the *ARTORG Center for Biomedical Engineering, University of Bern, Bern, Switzerland; +Graduate School for Cellular and Biomedical Sciences, University of Bern, Switzerland; and \#Department of Nephrology, Hypertension and Clinical Pharmacology, University of Bern, Inselspital, Bern, Switzerland.

Submitted for consideration June 2013; accepted for publication in revised form August 2013.

Disclosure: The ports were provided by Cendres + Métaux SA, Biel, Switzerland. Ethical approval was not required. The authors have no conflicts of interest to report.

This work was partly funded by a grant from the Swiss Commission for Technology and Innovation (CTI 13753.1).

Reprint Requests: Justyna Czerwinska, University of Bern, ARTORG Center, Biomedical Engineering Research, Murtenstrasse 50, Bern, Switzerland. Email: justyna.czerwinska@artorg.unibe.ch.

Copyright (C) 2013 by the American Society for Artificial Internal Organs

DOI: 10.1097/MAT.0000000000000015
Another alternative way for permanent vascular access is the implantation of a percutaneous dialysis port. Such a port is typically connected to a central vein via a catheter and can be repeatedly connected to the dialysis machine through a valve system..$^{1-3}$

In our institution, we are working on the development of a bone-anchored dialysis port. The general principle of this port is the fixation in bone of a titanium casing that permanently protuberates out of the skin. The titanium casing encloses a valve system that allows to connect a dialysis machine to a CVC via a detachable connection tubing set (Figure 1C). This approach is based on the long history of bone-anchored percutaneous implants for hearing loss therapy, ${ }^{4}$ cranio-facial surgery, ${ }^{5}$ or power supply to internal implants ${ }^{6}$ that show low complication rates. A more detailed description of the port's principle can be found in patent applications. ${ }^{7}$ Bone anchoring might give an alternative solution to address the frequent problems of bacterial migration encountered with previous port designs.

The design of such ports raises several challenges. The material properties of equipment designed to be in contact with circulating blood have been previously discussed. ${ }^{8}$ The management of the port-to-skin interface has also been explored..$^{9,10}$ This work focuses on the fluid dynamic aspects.

High pressure differences within a vascular access can eventually lead to hemolysis. ${ }^{11}$ The wall shear stress is cited as possible factor associated with vascular access complications. ${ }^{12}$ The effect of shear stress is related to the duration of the blood exposure to stress, hence the relevance of shear rate. The physiological range of shear rate is 1,000-10,000 $\mathbf{s}^{-1}$ and shear rates into the vascular system above $10,000 \mathbf{s}^{-1}$ are considered pathological. ${ }^{13}$ Another aspect is the possibility of blood platelet and complement activation resulting in thrombosis and stagnation of blood flow within the structure.

The design of a vascular access should allow high blood flow rate while keeping the pressure difference low and the local velocity and pressure in the physiological range. We explored two dialysis port designs and the potential impact of the lumen geometry inside the port on the induced pressure drop as well as on the shear rate.

The relationship between blood flow and pressure difference has been measured in vivo in various commercially available hemodialysis catheters. ${ }^{14}$ The isolated pressure drop induced by three catheter connectors: BD Q-Syte, Tego, and Codan Swan-LockU, were measured in vitro with water as a test fluid. ${ }^{15}$

A computer model of blood flow was reported for various catheter tip designs; straight cut, angled cut, and sleeve cut. The results report shear rates up to $13,000 \mathbf{s}^{-1}$ in all three measured catheters and stagnation zones in the angle and sleeve cut designs. ${ }^{16}$ The two catheters used in this study have split tips designs ended by a straight cut and a side hole. 
A

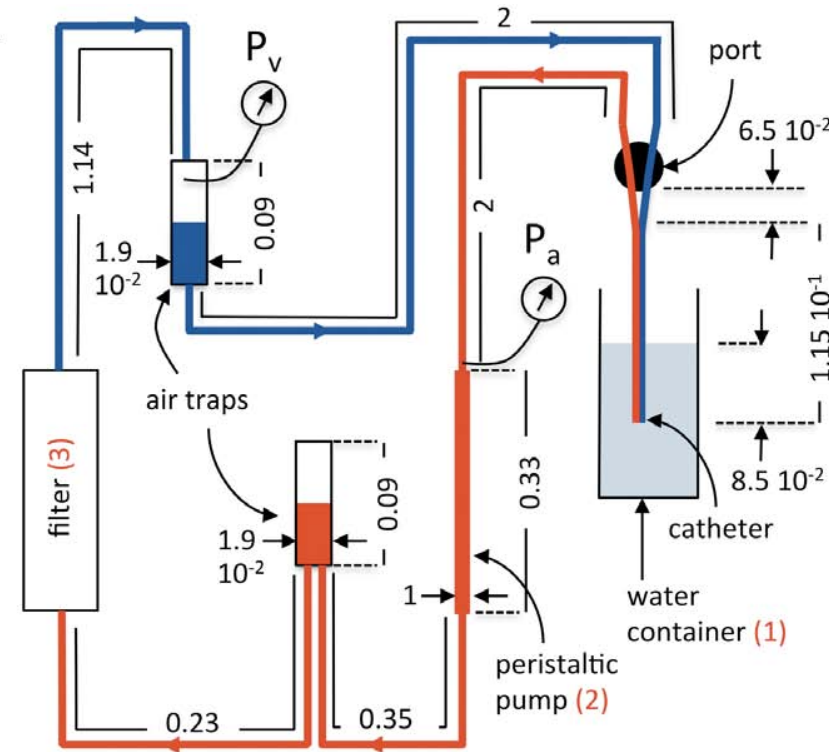

B

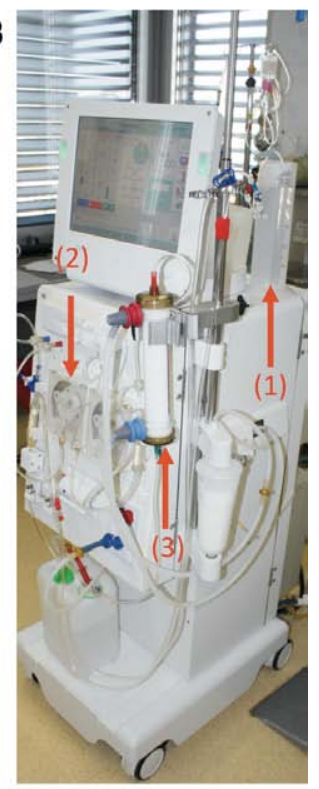

C

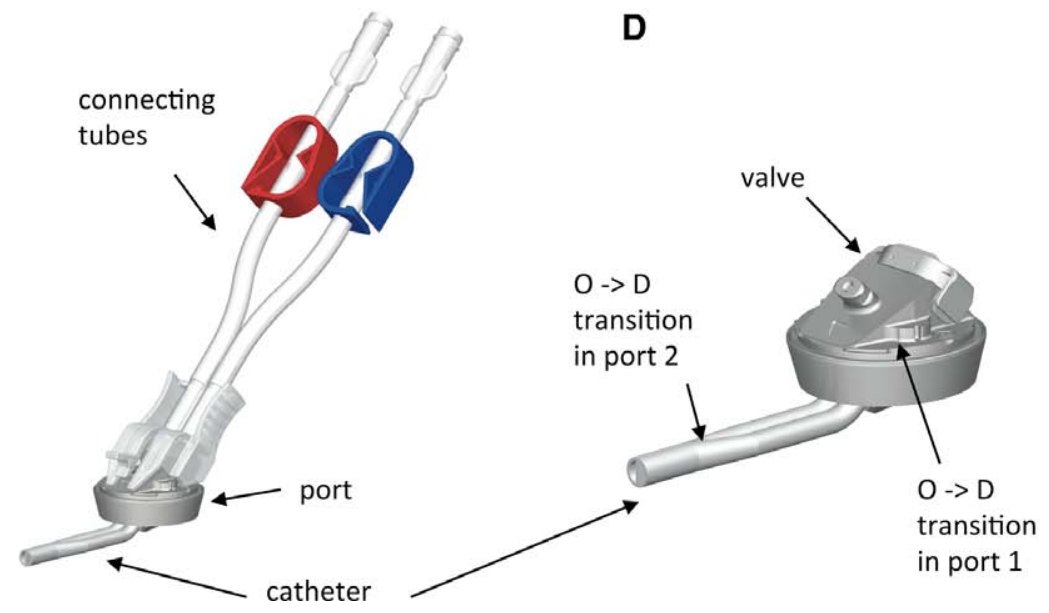

Figure 1. A: Schematic representation of the measurement setup: $P_{v}$ and $P_{a}$ are the hydrostatic pressure gauges for the venous/blue and arterial/red pressures. All dimensions are in meters. B: Picture of the measurement setup: (1) water container; (2) peristaltic pump; and (3) filter. C: Drawing of the connected port. D: Disconnected port-2. The location of the lumen's shape change is indicated for the two ports.

\section{Methods}

Flow Rate

A study on 160 patients reports clinical flow rates between 225 and $300 \mathrm{ml} / \mathrm{min}^{17}$ Other studies report blood flow rates between 350 and $419 \mathrm{ml} / \mathrm{min} .{ }^{12,18,19}$ We consequently decided to explore flow rates between 100 and $500 \mathrm{ml} / \mathrm{min}$ $\left(1.67-8.33 \times 10^{-6} \mathrm{~m}^{3} / \mathrm{s}\right)$ with steps of $50 \mathrm{ml} / \mathrm{min}$.

\section{Pressure Drop Measurements}

We conducted the measurements with water to be able to compare our results with the pressure drop induced by bloodline connectors reported in the literature. ${ }^{15}$

Two standard commercial hemodialysis catheters were used; the HemoStar (BARD Access Systems, Salt Lake City, UT) and the SplitStream (Medcomp, Harleysville, PA). With each of these catheters, a custom-built port prototype was used. The exact geometry of these ports is protected by intellectual property and cannot be reproduced in this work. The information relevant to the interpretation of the results is that both catheters have a transition from a round-shaped lumen at the dialysis machine side to a D-shaped lumen at the intravenous side. The main difference between the two tested ports is that in port- 1 this shape transition occurs within the port whereas in port-2 it occurs outside the port.

To isolate the contribution of separate elements of the portcatheter system in both designs, the following systems were investigated: an intact catheter with its standard connector; a catheter with its tip cut but with a standard connector; and two catheters with their tip cut connected to the two ports, replacing the standard connectors. The isolated pressure drop of the different elements was obtained by subtracting the pressure drop observed with a catheter with standard connector and tip cut from the observed pressure drops with the other configurations.

The setup was connected to a Dialog+ dialysis machine (B. Braun, Melsungen, Germany). A water container of $1,000 \mathrm{ml}$ was used as a patient substitute. The built-in hydrostatic 


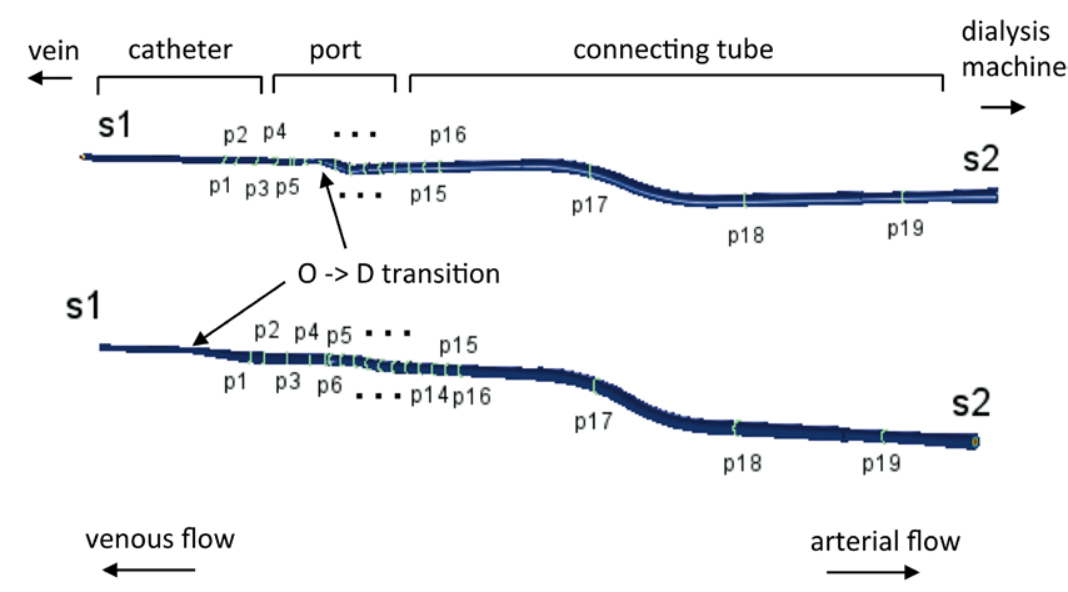

Figure 2. Models of the lumens along the ports: top: port-1, bottom: port-2. The lines indicate the cuts chosen for the analysis. The labels correspond to the specific two-dimensional cut definition. The location of the transition from round $(\mathrm{O})$ to $\mathrm{D}$-shaped lumen is indicated.

pressure sensors of the machine were used to measure the dynamic inflow pressure before the roller pump (arterial pressure) and the dynamic outflow pressure after the filter (venous pressure). See Figure 1 for an illustration and a schema of the setup.

To standardize the hydrostatic pressure at the catheter tip, the immersion depth of the tip, the water content, and the height of the container relatively to the pressure sensors were kept constant for all measurements. The pump flow rate was varied from 100 to $500 \mathrm{ml} / \mathrm{min}\left(1.67-8.33 \times 10^{-6} \mathrm{~m}^{3} / \mathrm{s}\right)$ in $50 \mathrm{ml} / \mathrm{min}$ steps.

\section{Data Acquisition and Analysis}

Measurements of pressure were averaged over 1 minute window by the built-in monitoring system. To ensure that the flow is in steady state, the measurements were only taken after the average flow rate over 1 minute stabilized to the specified peristaltic pump flow rate value. The manufacturer claims an accuracy of $2 \mathrm{mmHg}$ (267 Pa) for the pressure sensors.

The inlet Reynolds number was defined as $\operatorname{Re}=D v \rho / \mu$, where $D$ is the diameter of the lumen, $\rho$ the water density, $v$ the mean fluid velocity, and $\mu$ the dynamic viscosity of water. The Reynolds number is in the turbulence transition regime and the transition can be influenced by the surface roughness. We repeated the measurements five times with the same port design but different specimen and observed a negligible error in the pressure measurements. Therefore, the influence of the variability of surface roughness on the flow structures was estimated as insignificant.

The measurements errors of the pressure estimation and mass flow rate were $\delta p= \pm 1,333 \mathrm{~Pa} ; \delta Q= \pm 4.14 \times 10^{-7} \mathrm{~m}^{3} / \mathrm{s}$, respectively. For blood, the kinematic viscosity is $2.8 \times 10^{-6} \mathrm{~m}^{2} / \mathrm{s}$ and for water $10^{-6} \mathrm{~m}^{2} / \mathrm{s}$. Hence the Reynolds numbers reported for the same flow conditions will be approximately three times smaller in blood flow, but still within the turbulence transition regime. Hence, the main flow characteristics and flow patterns will change not in a large extent.

The pressure drop to flow rate relationship was fitted to the exponential equation for turbulence transition regime: $d p=A v^{B}$, where $d p$ is the pressure drop, $v$ is the flow velocity, and $A$ and $B$ are the fitted values.

\section{Finite Element Simulation}

Three-dimensional (3D) drawings of the port, the dialysis connector, and a portion of the catheter were created for both
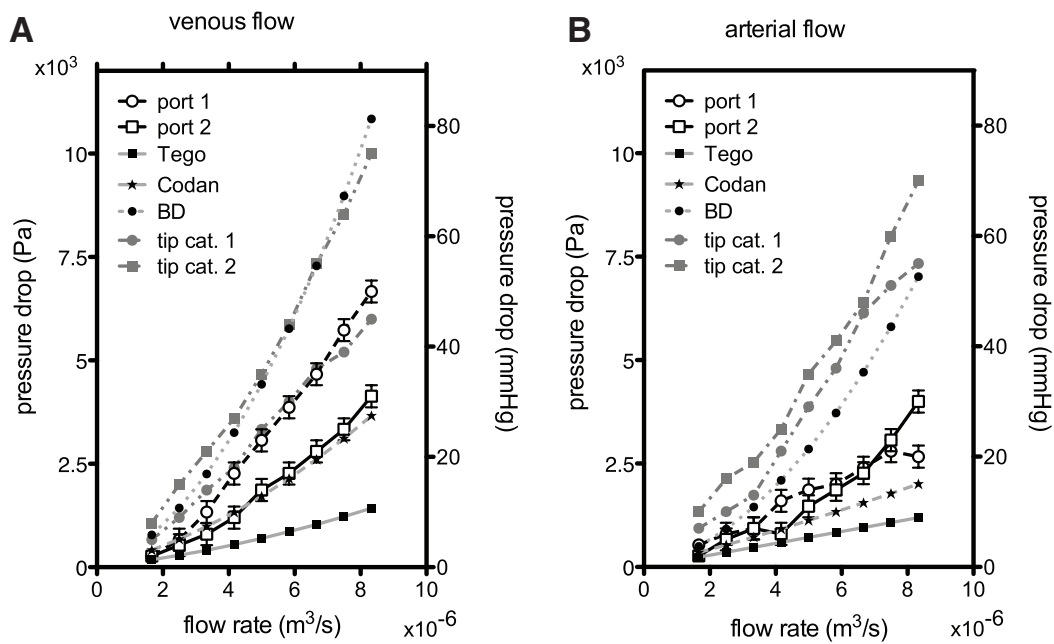

Figure 3. The isolated pressure drop induced by the two ports is compared, along with the tips of the catheters and commercially available catheter connectors reported in the literature. ${ }^{15} \mathbf{A}$ : venous flow; $\mathbf{B}$ : arterial flow. 


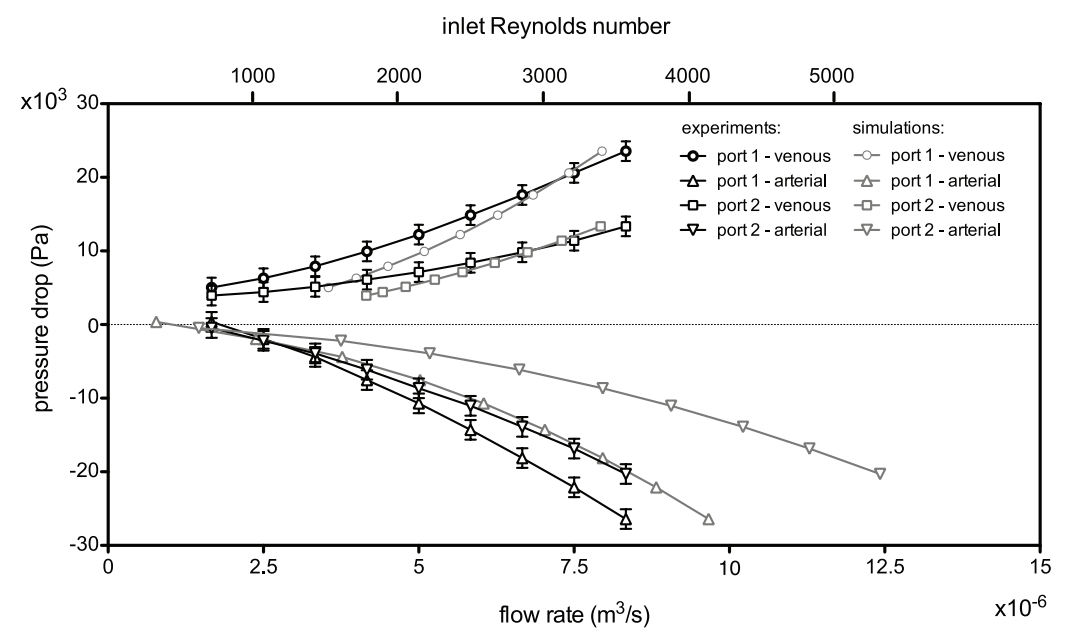

Figure 4. Computed (solid gray lines) vs. measured (solid black lines) pressure drop and volumetric flow rate relationship for both ports in both flow directions. The corresponding inlet Reynolds number is shown on the top scale.

port designs using SolidWorks (Dassault Systèmes SolidWorks Corp., Waltham, MA). Numerical simulations were carried out using COMSOL 4.3 (Comsol, Inc., Burlington, MA). Finite element simulations were performed, and after the mesh study, a mesh of more than 2 million tetrahedral elements was used. The mesh was created on the basis of the $3 \mathrm{D}$ drawings. The Navier-Stokes equations were used for flow description with the inlet pressure calibrated to the respective inlet experimental data. The pressure at the inlet and outlet are given. A Newtonian fluid with the density $\left(10^{3} \mathrm{~kg} / \mathrm{m}^{3}\right)$ and viscosity $\left(10^{-6} \mathrm{~m}^{2} / \mathrm{s}\right)$ of water was used as a medium. The boundary conditions were set according to the observations made in the experiments. To obtain the boundary conditions, the pressures at the inlet and outlet of the system represented in the 3D drawing was calculated analytically on the basis of the measurement setup (Figure 1) and on laminar flow theory in a pipe. The atmospheric pressure was set as a reference pressure. The velocity profiles of a selection of cuts were extracted. The locations of the intersections are marked on the lumens in Figure 2.

\section{Results}

The measured pressure drop relative to volume flow rate for the two ports is shown in Figure 3A for venous flow and Figure 3B for arterial flow. The pressure drop of a catheter was subtracted from the pressure drop of a catheter connected to the port to obtain the pressure drop of the port alone. The isolated effect of the catheter tips represented approximately $60 \%$ of the total catheters pressure drop. The port resulted in an increase of $20 \%$ in pressure drop compared with the catheter without port. The results plotted represent pressure drops of the port itself. This allows comparison of our results with reported results from the literature. The pressured drop induced by three commercial catheter connectors is also shown in Figure 3. The pressure drop in port- 1 was comparable with the one in port- 2 for arterial flow. However, it was on average 34\% higher for venous flow. The pressure drop in port- 2 was similar to the Codan connector in both directions and less than half of the BD connector. The pressure drop in port-2 was approximately $45 \%$ higher than in the Tego connector, for both directions.

Figure 4 shows both experimental and simulated relationships between pressure drop and flow rate in the two ports along with the respective inlet Reynolds number. The simulated values were similar to the measured values for venous flow. For arterial flow, the relative difference was similar in experimental and simulated data, but the simulations showed a smaller pressure drop than the measurements for a given flow rate. The Reynolds number was above 1,000 for most of the tested and simulated situations. This indicates that the observed flow is as expected, within the turbulence transition regime. The values of the exponential fit of the pressure and flow velocity relation: $d p=A v^{B}$ in both experiments and simulations are reported in Table $\mathbf{1 .}$

The velocity profiles at several cuts of port- 1 are shown in Figure 5. The pressure difference was set at the middle of the tested range. The velocity profiles for arterial flow for various inlet-outlet pressure difference at several cross-sections in port-1 is show in Figure 6. It can be seen that with the increase of the pressure difference, the transition to turbulence (characterized by large coherent structures) progresses further into the catheter. Figure 7 shows the velocity profiles for venous and arterial flow in port-2 through several cuts. The velocity profiles for arterial flow for various inlet-outlet pressure difference and several cross-sections for port-2 is drawn in Figure 8. It can be seen that with the increase in pressure difference,

Table 1. Exponential Fit of the Pressure and Flow Velocity Relation: $d p=A v^{B}$

\begin{tabular}{|c|c|c|c|c|c|c|c|c|}
\hline \multirow[b]{3}{*}{$\begin{array}{l}\text { Port } \\
\text { B }\end{array}$} & \multicolumn{4}{|c|}{ Simulations } & \multicolumn{4}{|c|}{ Experiments } \\
\hline & \multicolumn{2}{|c|}{ Venous } & \multicolumn{2}{|c|}{ Arterial } & \multicolumn{2}{|c|}{ Venous } & \multicolumn{2}{|c|}{ Arterial } \\
\hline & $\begin{array}{c}1 \\
1.91\end{array}$ & $\begin{array}{c}2 \\
1.89\end{array}$ & $\begin{array}{c}1 \\
1.72\end{array}$ & $\begin{array}{c}2 \\
1.89\end{array}$ & $\begin{array}{c}1 \\
0.99\end{array}$ & $\begin{array}{c}2 \\
0.77\end{array}$ & $\begin{array}{c}1 \\
2.19\end{array}$ & $\begin{array}{c}2 \\
2.54\end{array}$ \\
\hline
\end{tabular}

A value (B) close to 1 corresponds to the laminar flow. Higher values of B are present in turbulence transition. 


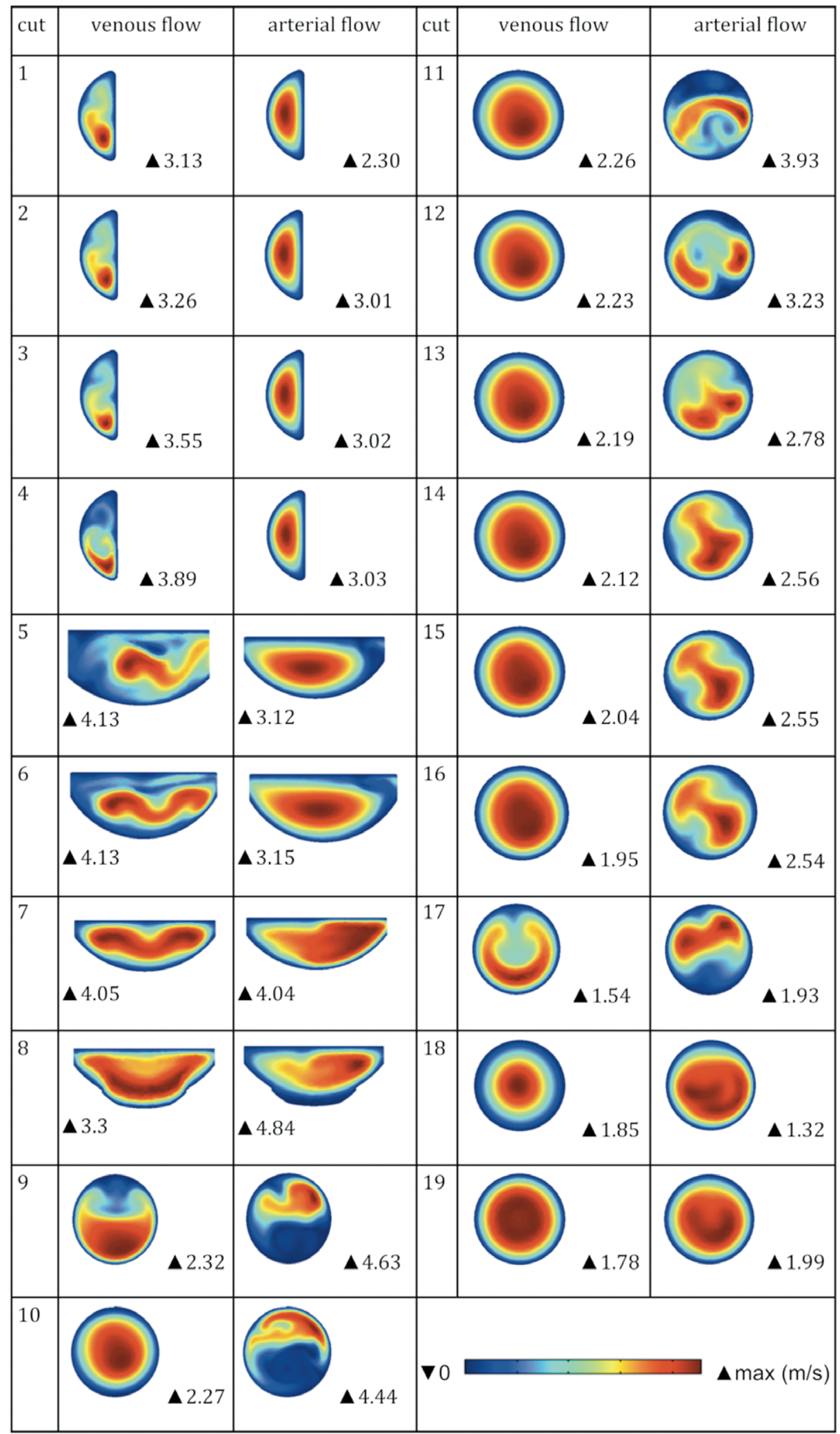

Figure 5. Venous $(\mathrm{dp}=13,238 \mathrm{~Pa})$ and arterial $(\mathrm{dp}=-9,700 \mathrm{~Pa})$ flow velocity profiles at various spatial cuts of port-1. For a description of cuts, see Figure 2.

the transition to turbulence progresses further into the catheter. The computed shear rate in the two ports for both arterial and venous flow is drawn in Figure 9. The maximal shear rate is approximately $8,000 \mathbf{s}^{-1}$ for the tested flow rates. 


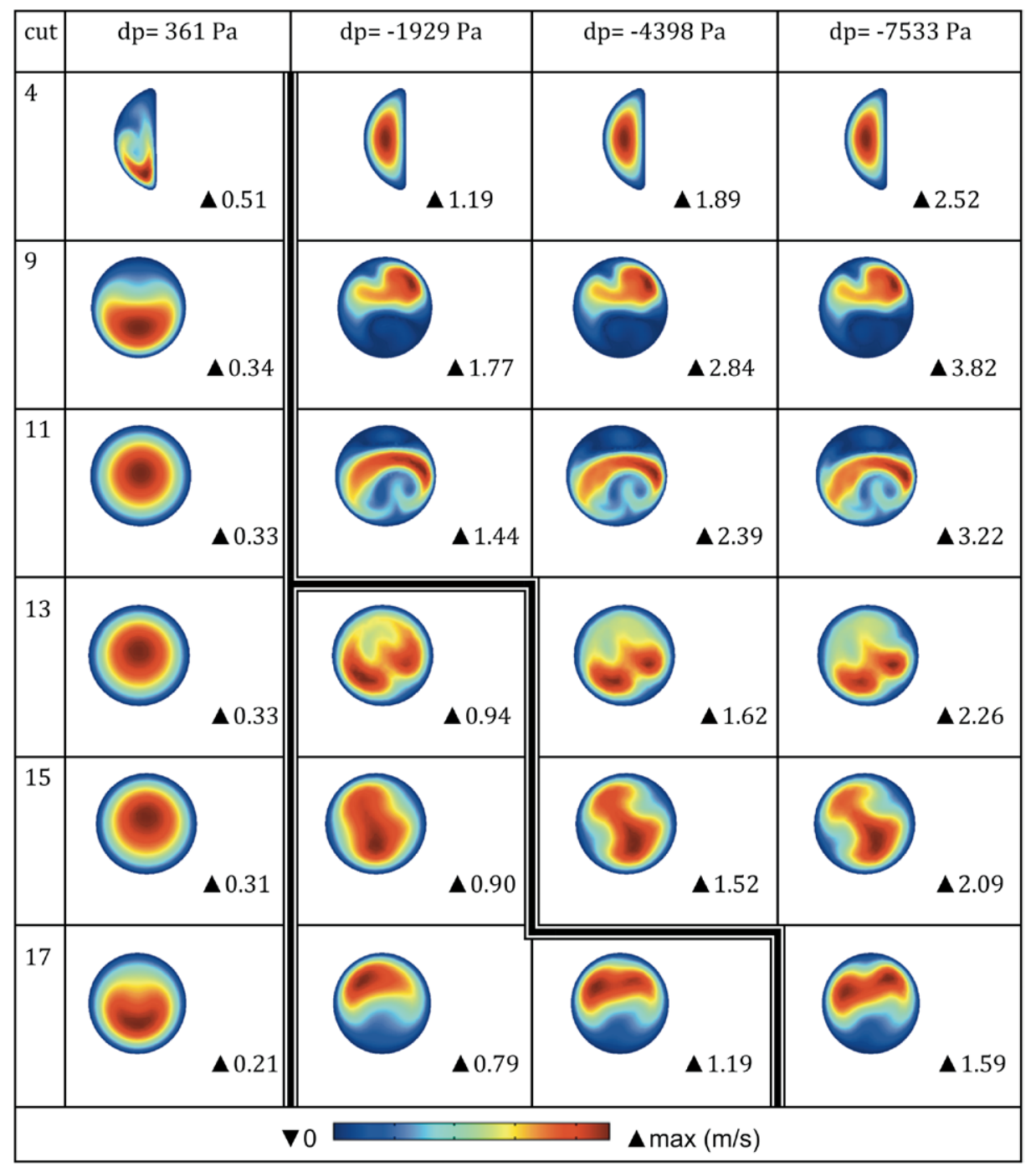

Figure 6. Arterial velocity flow profiles for arterial flow for various inlet-outlet pressure differences at several cross-sections of port-1. The bold line limits the region where the flow structures are changing.

\section{Discussion}

We have performed experimental and theoretical studies to answer several questions related to the port design, its compatibility, and possible advantages or drawbacks. First, we have measured pressure drops in relation to volumetric flow rates and compared these data with existing devices currently used in clinical routine. Figure 3 presents the values of the two investigated ports and corresponding data available from the literature. It can be seen that both investigated ports work in the range of laminar to turbulence transition. The Reynolds number was above 1,000 in most of the experiments and simulations (Figure 4). The values for venous flow through port- 1 and port- 2 show a good match between experiments and simulations. The simulations of arterial flow resulted in higher Reynolds numbers than the experiments, probably as a result of turbulence transition estimation. In the experiments, the walls are not perfectly smooth and that leads to a different development and type of flow structure as compared with the simulations based on ideally smooth walls. For a given Reynolds number, the pressure drop in experiments will be larger because of the port wall imperfections. The same conclusion can be drawn from Figure $\mathbf{4}$ and Table 1. The plots represent pressure drops as a function of the flow rate or velocity. Smooth walls in simulations lead a similar turbulence transition patterns in the direct (venous) and the reverse (arterial) flow. This can be seen especially well in Table 1, which gives fitting coefficients. In the case of simulations, both flow directions are of the same order in well-developed turbulence transition regime. For the experiments, a large difference can be observed. One direction is almost laminar (venous flow) and the other has highly developed turbulence regions (arterial flow). This can be a result of several artifacts. First, the pressure at the outlet and inlet of the water container was assumed to be purely hydrostatic. For high flow rates, this assumption is not true. There is definitely a flow within the water container, but we have had no means to estimate it. The container serves as representation of a patient on dialysis. In real life, in- and outflow parameters will greatly vary as well. If the average of the coefficients of venous and arterial flows is taken into account, they are closer to those of the simulation. The turbulence transition regime is very sensitive to the initial 


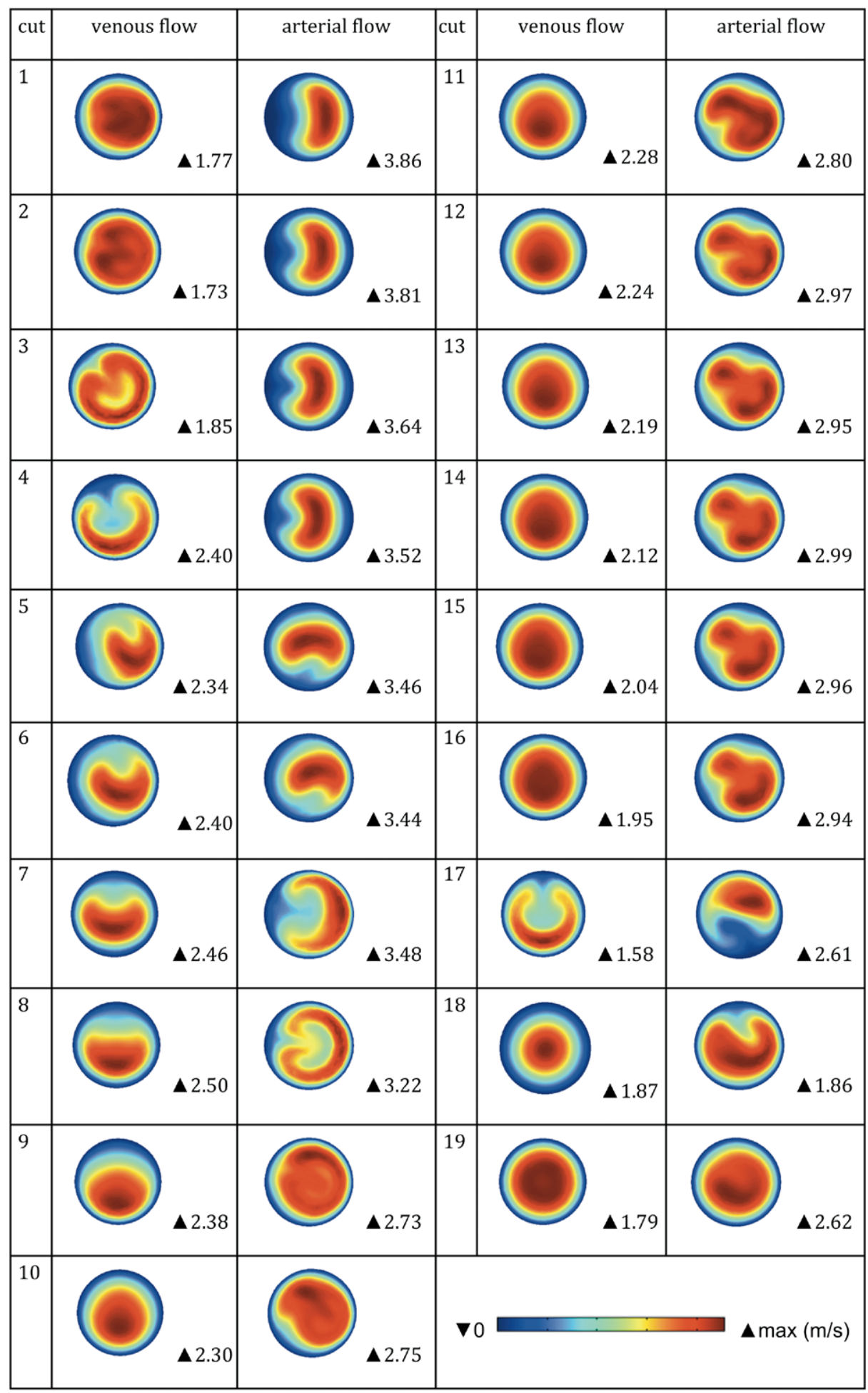

Figure 7. Venous $(\mathrm{dp}=7,971)$ and arterial $(\mathrm{dp}=-7,801 \mathrm{~Pa})$ velocity flow profiles at various spatial cuts of port-2. For a description of cuts, see Figure 2.

conditions and wall-fluid interaction. Hence, the exact values are very difficult to predict. However, the results obtained here are within the acceptable error.

The presented simulations allow a better and more detailed understanding of the specific characteristics of the ports working regime. The turbulence transition will lead to vortex formation in certain parts that could theoretically lead to blood hemolysis. Figure $\mathbf{5}$ shows the velocity of the venous $(\mathrm{dp}=13,238 \mathrm{~Pa})$ and arterial flow $(\mathrm{dp}=-9,700 \mathrm{~Pa})$ at all two-dimensional (2D) cross-sections. For venous flow, vortex structures are observed at cuts 1-9 and 17 and arterial ones at cuts 17 and 8 . Figure 6 shows arterial flow velocity for port- 1 


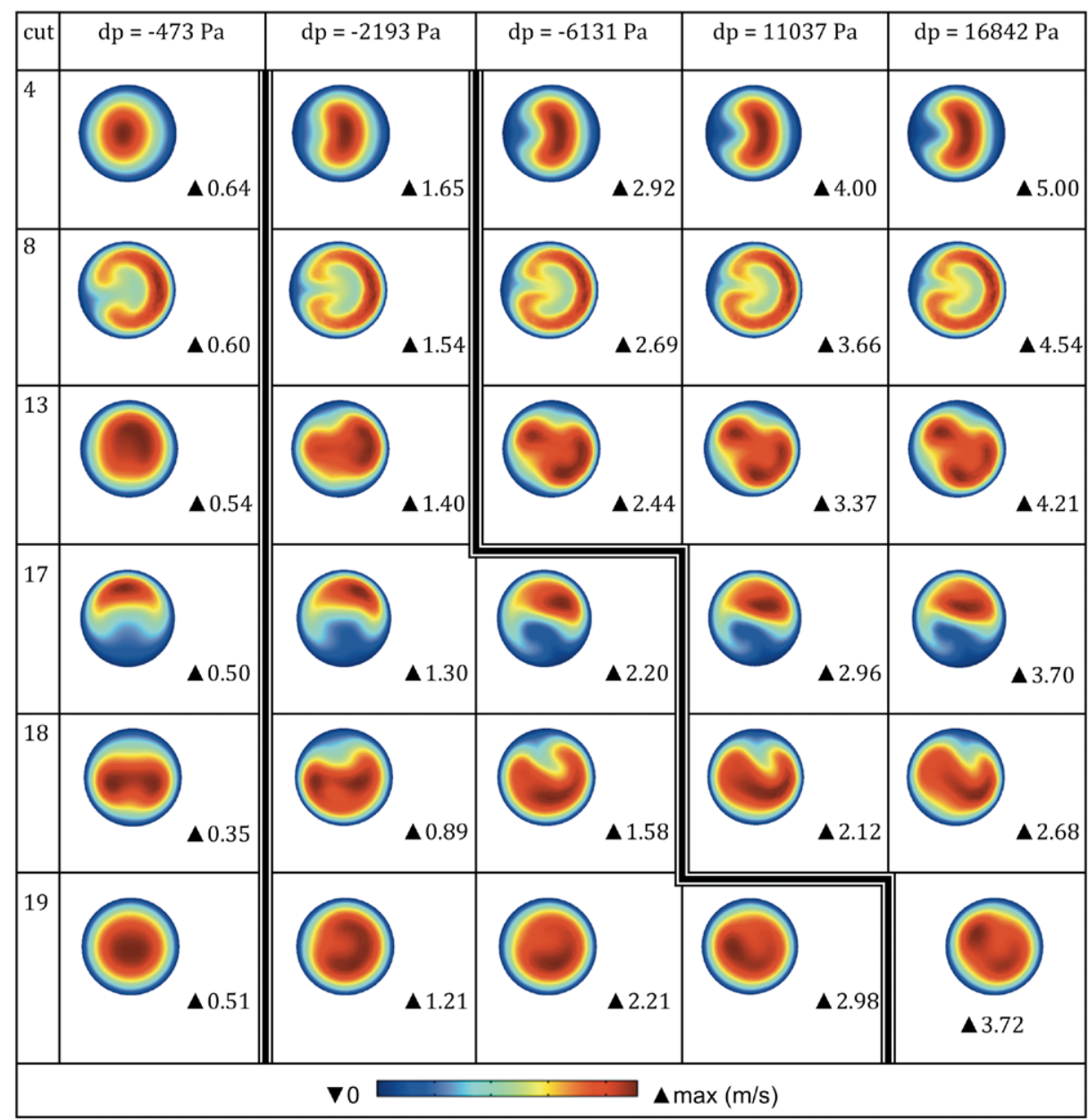

Figure 8. Velocity profiles for arterial flow for various inlet-outlet pressure difference and several cross-sections for the port-2. The bold line limits the region where the structures change.

at various pressure differences. Here, the transition to turbulence is more complex. The bold black line divides different

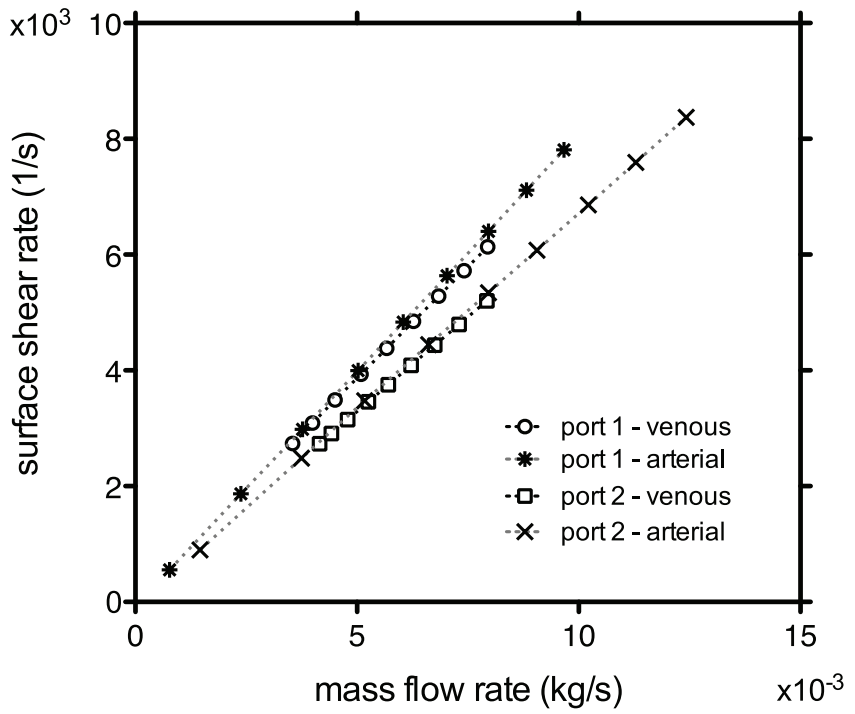

Figure 9. The computed shear rates as a function of mass flow rate in the two port prototypes both for venous (dotted black lines) and arterial (dotted grey lines) flow. flow regimes (laminar, first and second type of turbulence transition). The complexity of the turbulence transition could also explain the discrepancy between the two flow directions in the experiments. The complex vortex pattern always depends strongly on the surface, and if one flow direction is more prone to produce vortices, it will be more influence by wall impurities. Figure 7 shows velocity profiles for the crosssections of port-2 for venous flow $(\mathrm{dp}=7,971 \mathrm{~Pa})$ and arterial flow $(\mathrm{dp}=-7,801 \mathrm{~Pa})$. Venous flow shows vortex structures at cuts $3-8$ and 17 . For the arterial flow, almost all flow paths show some vortices. Hence, port-1 and port-2 vary significantly with respect to the arterial flow pattern and the way to the turbulence. Figure 8 shows the same quantities for arterial flow for port-2. It can be noted that again there are three regimes: fully laminar and first and second type of turbulence transition (shown by bold line dividing regions). The transition is present at a wider range of the whole geometry. Port- 1 has a narrowing at one end; consequently the vortex patterns were limited by viscous interactions. This is not the case for port-2. Hence, the transition vortex structures are more pronounced. The venous flow is definitely more efficient in port-2 because of the channel cross-section geometry. When a vortex pattern is present, it will be more stable in a larger cross-section than in a narrower one (port-1). However, the 
magnitude of the vortices differs as well. Port- 1 has approximately $50 \%$ stronger vortices for the same pressure difference as compared with port-2. Hence, the results for the global shear rate, plotted in Figure 9, are larger in port-1 (up to 15\%) for both flow directions. Globally this would mean that port- 1 would be more prone to blood hemolysis than port-2.

\section{Conclusion}

We have performed experiments and simulations to analyze the behavior of two newly designed dialysis ports. Both ports work in the turbulence transition regime, similar to conventional catheter connectors available on the market. However, the turbulence transition results in complex flow patterns with various vortices and therefore increases shear rate locally. The two proposed port designs vary in cross-section areas at a few points. This leads to different flow pattern characteristics, different ways of turbulence transition, approximately a $15 \%$ difference in the global shear rate values, and a 50\% difference in vortex strength.

The pressure drop induced by the two ports is comparable with commercial needle-less catheter connectors. The design with a round to D-shape transition outside the port is favorable over the one where it happens inside the port because the situation where the change in cross-section shape happens close to the sharp angle present in the port is more prone to turbulence. The finite elements model presented can be used for further development of the port. Following the results presented in this article, we chose to keep design number 2 for the clinical study.

\section{References}

1. Sandhu J: Dialysis ports: A new totally implantable option for hemodialysis access. Tech Vasc Interv Radiol 5: 108-113, 2002.

2. Prosl F, Polaschegg HD: New hemodialysis access system. Blood Purif 35: 72-76, 2013.

3. Canaud B, My H, Morena M, et al: Dialock: A new vascular access device for extracorporeal renal replacement therapy. Preliminary clinical results. Nephrol Dial Transplant 14: 692-698, 1999.

4. Tjellström A, Håkansson B: The bone-anchored hearing aid. Design principles, indications, and long-term clinical results. Otolaryngol Clin North Am 28: 53-72, 1995.
5. Gentie P, Bottini DJ, Gravante G, Nicoli F, Caruso R, Cervelli V: The use of bone-anchored implants for absent ear. / Craniofac Surg 19: 744-747, 2008.

6. Westaby S, Jarvik R, Freeland A, et al: Postauricular percutaneous power delivery for permanent mechanical circulatory support. J Thorac Cardiovasc Surg 123: 977-983, 2002.

7. Häusler R, Frey F, Stieger C, Arnold A, Farese S, Uehlinger D: Implantable access for removal and/or return of fluids. US Patent appl. $n^{\circ} 12 / 859,370,2011$.

8. Spijker HT, Graaff R, Boonstra PW, Busscher HJ, van Oeveren W: On the influence of flow conditions and wettability on blood material interactions. Biomaterials 24: 4717-4727, 2003.

9. Pendegrass CJ, Gordon D, Middleton CA, Sun SNM, Blunn GW: Sealing the skin barrier around transcutaneous implants. In vitro study of keratinocyte proliferation and adhesion in response to surface modification of titanium alloy. J Bone Joint Surg Br 90B: 114-121, 2008.

10. Smith CM, Roy TD, Bhalkikar A, Li B, Hickman JJ, Church KH: Engineering a titanium and polycaprolactone construct for a biocompatible interface between the body and artificial limb. Tissue Eng Part A 16: 717-724, 2010.

11. Twardowski ZJ, Haynie JD: Measurements of hemodialysis catheter blood flow in vivo. Int J Artif Organs 25: 276-280, 2002.

12. Tricht IV, Wachter DD, Tordoir J, Verdonck P: Hemodynamics and complications encountered with arteriovenous fistulas and grafts as vascular access for hemodialysis: A review. Ann Biomed Eng 33: 1142-1157, 2005.

13. Nesbitt WS, Westein E, Tovar-Lopez FJ, et al: A shear gradientdependent platelet aggregation mechanism drives thrombus formation. Nat Med 15: 665-673, 2009.

14. Ash SR, Mankus RA, Sutton JM, Spray M: The Ash Split CathTM as long-term IJ access: Hydraulic performance and longevity. I Vasc Access 3: 3-9, 2002.

15. Eloot S, De Vos JY, Hombrouckx R, Verdonck P: How much is catheter flow influenced by the use of closed luer lock access devices? Nephrol Dial Transplant 22: 3061-3064, 2007.

16. Mareels G, Kaminsky R, Eloot S, Verdonck PR: Particle image velocimetry-validated, computational fluid dynamics-based design to reduce shear stress and residence time in central venous hemodialysis catheters. ASAIO J 53: 438-446, 2007.

17. Schiffl H, Lang SM, Fischer R: Daily hemodialysis and the outcome of acute renal failure. N Engl J Med 346: 305-310, 2002.

18. Sherman RA, Matera JJ, Novik L, Cody RP: Recirculation reassessed: The impact of blood flow rate and the low-flow method reevaluated. Am J Kidney Dis 23: 846-848, 1994.

19. Atherikul K, Schwab SJ, Conlon PJ: Adequacy of haemodialysis with cuffed central-vein catheters. Nephrol Dial Transplant 13: 745-749, 1998. 